\title{
General very special relativity is Finsler geometry
}

\author{
G. W. Gibbons, ${ }^{1}$ Joaquim Gomis, ${ }^{2}$ and C. N. Pope ${ }^{3}$ \\ ${ }^{1}$ DAMTP, Centre for Mathematical Sciences, Cambridge University, Wilberforce Road, Cambridge CB3 OWA, United Kingdom \\ ${ }^{2}$ Departament ECM, Facultat de Física, Universitat de Barcelona, Diagonal 647, E-08028 Barcelona, Spain \\ ${ }^{3}$ George P. and Cynthia W. Mitchell Institute for Fundamental Physics, Texas A\&M University, \\ College Station, Texas 77843-4242, USA
}

(Received 20 August 2007; published 15 October 2007)

\begin{abstract}
We ask whether Cohen and Glashow's very special relativity model for Lorentz violation might be modified, perhaps by quantum corrections, possibly producing a curved space-time with a cosmological constant. We show that its symmetry group ISIM(2) does admit a 2-parameter family of continuous deformations, but none of these give rise to noncommutative translations analogous to those of the de Sitter deformation of the Poincaré group: space-time remains flat. Only a 1-parameter family $\operatorname{DISIM}_{b}(2)$ of deformations of $\operatorname{SIM}(2)$ is physically acceptable. Since this could arise through quantum corrections, its implications for tests of Lorentz violations via the Cohen-Glashow proposal should be taken into account. The Lorentz-violating point-particle action invariant under $\operatorname{DISIM}_{b}(2)$ is of Finsler type, for which the line element is homogeneous of degree 1 in displacements, but anisotropic. We derive $\operatorname{DISIM}_{b}(2)$-invariant wave equations for particles of spins $0, \frac{1}{2}$, and 1 . The experimental bound, $|b|<$ $10^{-26}$, raises the question "Why is the dimensionless constant $b$ so small in very special relativity?"
\end{abstract}

DOI: 10.1103/PhysRevD.76.081701

PACS numbers: 03.30.+p, 02.20.Sv, 11.30.Cp, 11.30.Er

Local Lorentz and $C P T$ invariance are fundamental assumptions in almost all current physical theories. It is important to test these assumptions experimentally, lest evidence of new physics beyond the standard model be overlooked. Current experimental limits on violations of local Lorentz and $C P T$ invariance are extremely stringent. Thus what is required are novel alternative non-Lorentz invariant theories, capable of circumventing these tight limits. Recently, Cohen and Glashow [1] have made the ingenious proposal that the local laws of physics need not be invariant under the full Lorentz group, generated by $M_{\mu \nu}$, but rather, under a $\operatorname{SIM(2)}$ subgroup, whose Lie algebra is generated by $\left(M_{+i}, M_{i j}, M_{+-}=M_{03}\right)$, (with $i$ and $j$ ranging over the values 1 and 2) [2]. This they referred to as very special relativity. Taking the semidirect product with the translations $\left(P_{+}, P_{-}, P_{i}\right)$ gives an 8dimensional subgroup of the Poincare group called $\operatorname{ISIM}(2)$ [3].

The great merits of Cohen and Glashow's suggestion are that $C P T$ symmetry is preserved and that $\operatorname{ISIM}(2)$ leaves invariant no vector or tensor fields, known as "spurion fields." For example, a spurionic vector field may be thought of as the 4-velocity of the æther [4]. In fact SIM(2) consists of those Lorentz transformations $\Lambda^{\mu}{ }_{\nu}$ leaving invariant the null direction $n^{\mu}=\delta_{+}^{\mu}$, i.e., such that $\Lambda_{\nu}^{\mu} n^{\nu}=\lambda n^{\mu}$ for some $\lambda$ which depends on $\Lambda^{\mu}{ }_{\nu}$. The generator $M_{+-}$acts by sending $n^{\mu} \rightarrow \lambda n^{\mu}$. This scaling symmetry implies that one cannot take $n^{\mu}$ to define the actual 4-velocity of the rthereal motion, but only its direction, thus rendering the presence of such an æther more difficult to detect. A theory of this kind appears to be compatible with all current experimental limits on violations of Lorentz invariance and spatial isotropy $[1,5]$.
Subsequently, Cohen and Freedman [6], and later Lindström and Roček [7], showed that ISIM is compatible with supersymmetry. There are several ways one might try to incorporate gravity. One is where we make local the ISIM(2) algebra [8]. Another is to consider a global spacetime and to see if it is compatible with very special relativity ideas. In this case there appear to be difficulties [9]. For example, the maximal subgroup of $S O(4,1)$, the

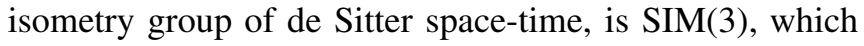
is 7-dimensional [10]. $\operatorname{SIM(3)~contains~} \operatorname{SIM}(2)$ as a subgroup, but the stabilizer, or tangent-space group, is $\mathrm{SO}(3)$, not $\operatorname{SIM}(2)$.

Alternatively, we recall that Poincare group admits a unique deformation into the de Sitter (anti-de Sitter) group [11], with $\left[P_{\mu}, P_{\nu}\right]=\frac{1}{3} \Lambda M_{\mu \nu}$, where the parameter $\Lambda$ is the cosmological constant. One may ask whether ISIM(2) admits a similar deformation, such that the translations $P_{\mu}$ become noncommutative. If so, the coset of the deformed group divided by $\operatorname{SIM}(2)$ (or its deformation) could be thought of as a curved space-time.

Here we show that there are indeed continuous deformations of $\operatorname{ISIM(2),~but~in~all~of~them~the~translations~}$ remain commutative. Among them is a 1-parameter family of deformations, which we denote by $\operatorname{DISIM}_{b}(2)$. For any values of $b$ this is an 8-dimensional subgroup of the 11dimensional Weyl group, i.e., the semidirect product of dilatations with the Poincaré group. Subgroups with different values of $b$ are not isomorphic. Interestingly, if one constructs a point-particle action for the deformed groups $\operatorname{DISIM}_{b}(2)$, using the methods of nonlinear realizations [12], one arrives at Lagrangians of Finsler form, first proposed by Bogoslovsky (see [13] and references therein). Therefore the deformation of very special relativ- 
ity leads in a natural way to Finsler geometry. In the remainder of this paper we shall outline the derivation of these results, and comment on their physical significance.

Continuous deformations of Lie algebras have been extensively explored, by both mathematicians and physicists, under the rubric of Lie-algebra cohomology [11]. Here we give an elementary account based on the Cartan-Maurer equations, which provides a simple and easily automated scheme for determining the deformations of a given Lie algebra $g$ with structure constants $C_{a}{ }^{b}{ }_{c}$. We suppose there exists a family of deformed Lie algebras $\mathfrak{g}_{t}$ parametrized by a continuous variable $t$, with structure constants

$$
\hat{C}_{a}{ }_{a}{ }^{b}{ }(t)=C_{a}{ }^{b}{ }_{c}+t A_{a}{ }^{b}{ }_{c}+t^{2} B_{a}{ }^{b}{ }_{c}+\cdots .
$$

We are only interested in deformations which do not arise merely from a ( $t$-dependent) change of basis:

$$
\hat{C}_{a}{ }_{a}{ }^{b}(t)=S^{b}{ }_{e} C_{d f}{ }^{e}\left(S^{-1}\right)^{d}{ }_{a}\left(S^{-1}\right)^{f}{ }_{c}, \quad S^{a}{ }_{b} \in G L(n, \mathbb{R}) .
$$

Expanding the Jacobi identity $\hat{C}_{\left.d{ }^{[}{ }^{e}(t) \hat{C}_{b}{ }^{d}\right]}(t)=0$ in powers of $t$ gives rise at linear order to

$$
C_{d[a}^{e} A_{b}{ }^{d}{ }_{c]}+A_{d[a}^{e} C_{b}{ }^{d}{ }_{c]}=0 .
$$

A first-order deformation $A$ will be trivial if $S^{a}{ }_{b}(t)=\delta_{b}^{a}+$ $t \Phi^{a}{ }_{b}+\cdots$ and

$$
A_{a}{ }^{b}{ }_{c}=\Phi^{b}{ }_{e} C_{a}{ }^{e}{ }_{b}-C_{e}{ }^{b}{ }_{c} \Phi^{e}{ }_{a}-C_{a}{ }^{b}{ }_{e} \Phi^{e} .
$$

Introducing a basis $\lambda^{a}$ of left-invariant 1-forms of the original algebra, such that $d \lambda^{a}=-\frac{1}{2} C_{b}{ }^{a}{ }_{c} \lambda^{b} \wedge \lambda^{c}$, we define vector-valued 1-forms and 2-forms $\Phi^{a} \equiv \Phi_{b} \lambda^{b}$ and $A^{a} \equiv \frac{1}{2} A_{b}{ }^{a}{ }_{c} \lambda^{b} \wedge \lambda^{c}$ and a matrix-valued 1-form $C^{a}{ }_{b} \equiv \lambda^{c} C_{c}{ }^{a}{ }_{b}$. Defining $D \equiv d+C \wedge$, the first-order deformation equations (3) may then be written as

$$
D A=0, \quad A \neq-D \Phi,
$$

where the second equation expresses the requirement of nontriviality of the deformation. Because $D^{2}=0$ as a consequence of the Jacobi identities of the undeformed algebra, $d C+C \wedge C=0$, the differential $D$ may be regarded as a coboundary operator acting on $\mathfrak{g}$-valued forms. The nontrivial linearized deformations are therefore in 1-1 correspondence with the second cohomology group $H^{2}(\mathfrak{g} ; \mathfrak{g})$.

If a nontrivial linear deformation $A$ is found, the next step is to investigate the Jacobi identities at order $t^{2}$. These read

$$
\left.C_{d[a}{ }^{e} B_{b}{ }^{d}{ }_{c]}+B_{d[a}^{e} C_{b}{ }^{d}{ }_{c]}+A_{d[a}{ }^{e} A_{b}{ }^{d}{ }_{c]}\right]=0,
$$

and can be reexpressed in terms of the vector and matrix valued forms as

$$
\begin{aligned}
D B+A \bullet A & =0, \\
(A \bullet A)^{e} & \equiv \frac{1}{2} A_{d}{ }^{e}\left[{ }_{a} A_{b}{ }^{d}{ }_{c} \lambda^{\lambda^{a}} \wedge \lambda^{b} \wedge \lambda^{c} .\right.
\end{aligned}
$$

This equation can only be solved if $D(A \bullet A)=0$, which implies that $A \bullet A$ must be both $D$ closed and exact. Thus there is a potential obstruction to finding a deformation at quadratic order: $A \bullet A$ should not have a projection in the third cohomology group $H^{3}(\mathfrak{g} ; \mathfrak{g})$. There are analogous equations to (7) at higher orders in $t$. If $H^{3}(\mathfrak{g} ; \mathfrak{g})$ vanishes, then the equations may be solved at all nonlinear orders. If $H^{3}(g ; \mathfrak{g})$ is nonzero, then the higher-order analogues of $(A \bullet A)$ should have no projections into it.

We start with the $\operatorname{ISIM}(2)$ Cartan-Maurer relations

$$
\begin{gathered}
d \lambda^{+}=\lambda^{+i} \wedge \lambda^{i}+\lambda^{+-} \wedge \lambda^{+}, \quad d \lambda^{-}=-\lambda^{+-} \wedge \lambda^{-} \\
d \lambda^{i}=\epsilon_{i j} \lambda^{12} \wedge \lambda^{j}+\lambda^{-} \wedge \lambda^{+i} \\
d \lambda^{+i}=\epsilon_{i j} \lambda^{12} \wedge \lambda^{+j}+\lambda^{+-} \wedge \lambda^{+i}, \quad d \lambda^{+-}=0 \\
d \lambda^{12}=0
\end{gathered}
$$

where $\quad g^{-1} d g=\lambda^{+} P_{+}+\lambda^{-} P_{-}+\lambda^{i} P_{i}+\lambda^{+i} M_{+i}+$ $\lambda^{+-} M_{+-}+\lambda^{12} M_{12}$. Defining $N \equiv M_{+-}$and $J \equiv M_{12}$, the corresponding nontrivial Lie brackets are therefore

$$
\begin{aligned}
& {\left[N, P_{ \pm}\right]=\mp P_{ \pm}, \quad\left[N, M_{+i}\right]=-M_{+i},} \\
& {\left[J, P_{i}\right]=\epsilon_{i j} P_{j}, \quad\left[J, M_{+i}\right]=\epsilon_{i j} M_{+i},} \\
& {\left[M_{+i}, P_{-}\right]=P_{j}, \quad\left[M_{+i}, P_{j}\right]=-\delta_{i j} P_{+} .}
\end{aligned}
$$

Expanding the vector-valued 2-form $A^{a}$ on a basis of 2forms and solving the resultant linear equations in (5) reveals that there is a 2-parameter family of nontrivial solutions, i.e., $H^{2}(\mathfrak{i} \mathfrak{i m}(2) ; \mathfrak{i m m}(2))$ is 2-dimensional. Substituting this linearized solution into the full Jacobi identities, we find that it gives a 2-parameter family of exact Lie algebras of the leading-order form (8), with additional terms as follows:

$$
d \lambda^{\mu} \rightarrow d \lambda^{\mu}+a \lambda^{12} \wedge \lambda^{\mu}+b \lambda^{+-} \wedge \lambda^{\mu},
$$

where $\mu=(+,-, i)$. Here $a$ and $b$ are arbitrary constant parameters.

As in the undeformed case, the algebra here has the structure of a semidirect sum of $\mathfrak{s i n}(2)$ and the translations $\mathbb{R}^{4}$, i.e., $\operatorname{gim}(2) \ltimes \mathbb{R}^{4}$. While the $M_{+i}$ act on the translations as in the undeformed case, the adjoint action of the generators $N$ and $J$ is given by $\left[N, P_{\mu}\right]=P_{\nu} C_{N}{ }^{\nu}{ }_{\mu}$ and $\left[J, P_{\mu}\right]=P_{\nu} C_{J}{ }^{\nu}{ }_{\mu}$, where the matrices $C_{N}$ and $C_{J}$ are given, respectively, by 


$$
\begin{gathered}
-\left(\begin{array}{cccc}
b+1 & 0 & 0 & 0 \\
0 & b-1 & 0 & 0 \\
0 & 0 & b & 0 \\
0 & 0 & 0 & b
\end{array}\right), \\
\left(\begin{array}{cccc}
-a & 0 & 0 & 0 \\
0 & -a & 0 & 0 \\
0 & 0 & -a & -1 \\
0 & 0 & 1 & -a
\end{array}\right)
\end{gathered}
$$

Minkowski space-time may be thought of as the symmetric space $E(3,1) / S O(3,1)$ with $S O(3,1)$ playing the role of the tangent-space group, and the tangent space being spanned by the translations $P_{\mu}$. In our case we wish to replace $S O(3,1)$ by $\operatorname{SIM}(2)$. However, it follows by exponentiating $C_{J}$ that $J$ does not generate a compact $S O(2)$ subgroup unless the deformation parameter $a$ vanishes. From now on we shall restrict attention to this $a=0$ case, for which we denote the deformed algebra by $\operatorname{Digim}_{b}(2)$.

The nontrivial Lie brackets for $\operatorname{Digim}_{b}(2)$ are given by

$$
\begin{gathered}
{\left[N, P_{ \pm}\right]=-(b \pm 1) P_{ \pm}, \quad\left[N, P_{i}\right]=-b P_{i},} \\
{\left[N, M_{+i}\right]=-M_{+i}, \quad\left[J, P_{i}\right]=\epsilon_{i j} P_{j},} \\
{\left[J, M_{+i}\right]=\epsilon_{i j} M_{+i}, \quad\left[M_{+i}, P_{-}\right]=P_{j},} \\
{\left[M_{+i}, P_{j}\right]=-\delta_{i j} P_{+} .}
\end{gathered}
$$

The deformed group $\operatorname{DISIM}_{b}(2)$ is a subgroup of the Weyl group with an action on Minkowski space-time given by translations, and boosts in the $+i$ directions, together with a combination of a boost in the +- direction and a dilatation. Specifically, the deformed generator $N$ acts as

$$
x^{i} \rightarrow \lambda^{-b} x^{i}, \quad x^{-} \rightarrow \lambda^{1-b} x^{-}, \quad x^{+} \rightarrow \lambda^{-1-b} x^{+} .
$$

The group DISIM ${ }_{b}(2)$ does not leave invariant the standard Minkowski line element $d s=\left(\eta_{\mu \nu} d x^{\mu} d x^{\nu}\right)^{1 / 2}$, but rather, the Finslerian line element

$$
\begin{aligned}
d s & =\left(2 d x^{+} d x^{-}+d x^{i} d x^{i}\right)^{(1-b) / 2}\left(d x^{-}\right)^{b} \\
& =\left(\eta_{\mu \nu} d x^{\mu} d x^{\nu}\right)^{(1-b) / 2}\left(n_{\rho} d x^{\rho}\right)^{b} .
\end{aligned}
$$

This is of the form first suggested by Bogoslovsky [13].

We shall now construct, using the theory of nonlinear realizations, a DISIM $(2)$-invariant Lagrangian for a point particle. We parametrize the coset $\operatorname{DISIM}_{b}(2) / S O(2)$ as

$$
g=e^{x^{\mu} P_{\mu}} e^{w^{i} M_{+i}} e^{w N},
$$

which implies that

$$
\begin{aligned}
g^{-1} d g= & d w N+e^{w} d w^{i} M_{+i}+e^{-w(1-b)} d x^{-} P_{-} \\
& +e^{w(1+b)}\left(d x^{+}+w^{i} d x^{i}-\frac{1}{2} w^{i} w^{i} d x^{-}\right) P_{+} \\
& +e^{w b}\left(d x^{i}-w^{i} d x^{-}\right) P_{i} \\
= & \lambda^{+-} N+\lambda^{+i} M_{+i}+\lambda^{-} P_{-}+\lambda^{+} P_{+}+\lambda^{i} P_{i},
\end{aligned}
$$

where $\left(\lambda^{+-}, \lambda^{+i}, \lambda^{+}, \lambda^{-}, \lambda^{i}\right)$ are the restrictions (or pullbacks) of the invariant 1 -forms on the group $\operatorname{DISIM}_{b}(2)$ to the coset $\operatorname{DISIM}_{b}(2) / S O(2)$.

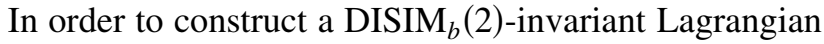
with worldline reparametrization invariance, we allow the Goldstone coordinates $\left(w, w^{i}, x^{\mu}\right)$ to depend on the worldline coordinate $\tau$ (see, for example, [14]). We shall restrict our attention to Lagrangians that are linear in the leftinvariant 1-forms pulled back to the particle's worldline. Requiring invariance under $S O(2)$ then implies that we must discard $\lambda^{+i}$ and $\lambda^{i}$, and thus we consider the Lagrangian

$$
\begin{aligned}
\mathcal{L}= & \alpha e^{w(1+b)}\left(\dot{x}^{+}+w^{i} \dot{x}^{i}-\frac{1}{2} w^{i} w^{i} \dot{x}^{-}\right)+\beta e^{-w(1-b)} \dot{x}^{-} \\
& +\gamma \dot{w},
\end{aligned}
$$

where $\alpha, \beta$, and $\gamma$ are arbitrary constants. Since the last term is a total derivative, we can discard it. Eliminating the nondynamical Goldstone coordinates $w$ and $w^{i}$, one obtains, in physical units, the Lagrangian

$$
\mathcal{L}=-m\left(-\eta_{\mu \nu} \dot{x}^{\mu} \dot{x}^{\nu}\right)^{(1-b) / 2}\left(-n_{\rho} \dot{x}^{\rho}\right)^{b} .
$$

Calculating the canonical momenta from (17), we obtain the $\operatorname{DISIM}_{b}(2)$-invariant dispersion relation or Hamiltonian constraint

$$
\eta^{\mu \nu} p_{\mu} p_{\nu}=-m^{2}\left(1-b^{2}\right)\left(-\frac{n^{\nu} p_{\nu}}{m(1-b)}\right)^{2 b /(1+b)} .
$$

Note that for $b=0$ we recover the ordinary free relativistic particle, which does not see the lightlike direction $n^{\mu}$. The cases $b= \pm 1$ are special, and are best investigated directly from Eq. (16). The conclusion is that for $b=1$ we obtain the massless equation $\eta^{\mu \nu} p_{\mu} p_{\nu}=0$, while for $b=-1$ we have $\dot{x}^{i}=0$ and $\dot{x}^{-}=0$, and the dynamics is trivial in this case.

Upon quantization, $p_{\mu} \rightarrow-\mathrm{i} \partial_{\mu}$, we obtain a generalized Klein-Gordon equation of the form

$$
-\square \phi+m^{2}\left(1-b^{2}\right)\left(\frac{\mathrm{i} n^{\mu} \partial_{\mu}}{m(1-b)}\right)^{2 b /(1+b)} \phi=0 .
$$

This is in general a nonlocal equation, since it involves fractional derivatives. Although the special case $b=1$ appears to give a local modification of the usual KleinGordon equation involving a term linear in $n^{\mu} \partial_{\mu}$ this is really equivalent to the standard massless Klein-Gordon equation (as discussed earlier for this special value of $b$ ). 
Specifically, the first-order term can be removed by making the phase transformation $\phi \rightarrow \phi e^{-\mathrm{i} m n_{\mu} x^{\mu} / 2}$.

The free Maxwell equations are also invariant under the action of the Weyl group and so they too are clearly invariant under $\operatorname{DISIM}_{b}(2)$. The invariance of $A_{\mu} d x^{\mu}$, together with (12), implies that $\left(A_{+}, A_{-}, A_{i}\right) \rightarrow$ $\left(\lambda^{b+1} A_{+}, \lambda^{b-1} A_{-}, \lambda^{b} A_{i}\right)$. Since $d^{4} x \rightarrow \lambda^{-4 b} d^{4} x$ any invariant action must have $\mathcal{L} \rightarrow \lambda^{4 b} \mathcal{L}$. Thus we can add a mass term, giving

$$
\mathcal{L}=-\frac{1}{4} F_{\mu \nu} F^{\mu \nu}-\frac{1}{2} m^{2}\left(\frac{\left(n^{\mu} A_{\mu}\right)^{2}}{A_{\nu} A_{\nu}}\right)^{b} A_{\rho} A^{\rho} .
$$

If $b=1$ we can further include a non-Lorentz invariant Chern-Simons term [15], $\mathcal{L}_{\mathrm{cs}}=\frac{1}{2} \ell^{-1} \epsilon^{\mu \nu \rho \sigma} n_{\mu} A_{\nu} F_{\rho \sigma}$, where $\ell$ is an arbitrary length scale.

Since it is a subgroup of the Weyl group, $\operatorname{DISIM}_{b}(2)$ leaves invariant the massless Dirac Lagrangian. Bogoslovsky and Goenner [16] have pointed out that adding to the massless Dirac Lagrangian a term of the form

$$
m\left[\left(\frac{\mathrm{i} n_{\mu} \bar{\psi} \gamma^{\mu} \psi}{\bar{\psi} \psi}\right)^{2}\right]^{b / 2} \bar{\psi} \psi
$$

gives a nonlinear $\operatorname{DISIM}_{b}(2)$-invariant generalization of the massive Dirac equation. This follows from the scalings $\psi \rightarrow \lambda^{3 b / 2} \psi, \gamma^{\mu} \partial_{\mu} \rightarrow \lambda^{b} \gamma^{\mu} \partial_{\mu}$ under the action of the generator $M_{+-}$. As with the generalized Klein-Gordon equation (19), the case $b=1$ is special: The additional term may then be removed by a phase transformation of the form $\psi \rightarrow \psi e^{\mathrm{i} m n_{\mu} x^{\mu}}$. Note however that, as discussed below, experimental bounds constrain $|b|$ to be very much less than 1.

$C P T$ will be preserved if an operator exists in the complexification of $\operatorname{DISIM}_{b}(2)$ that reverses $x^{\mu}$. As discussed for ISIM(2) in [1], a candidate $C P T$ operator is $e^{\mathrm{i} \phi J} e^{\mathrm{i} \alpha N}$. This has the following action on the momenta:

$\left(P_{+}, P_{-}, P_{1}+\mathrm{i} P_{2}\right) \rightarrow e^{-b \alpha}\left(e^{-\alpha} P_{+}, e^{\alpha} P_{-}, e^{\mathrm{i} \phi}\left(P_{1}+\mathrm{i} P_{2}\right)\right)$.

Requiring that $P_{\mu} \rightarrow-P_{\mu}$ implies that

$$
\begin{gathered}
\alpha=\mathrm{i} \pi\left(n_{-}-n_{+}\right), \quad \phi=\pi\left(2 n_{3}-n_{+}-n_{-}\right), \\
b=\frac{1+n_{+}+n_{-}}{n_{+}-n_{-}},
\end{gathered}
$$

for integers $n_{+}, n_{-}$, and $n_{3}$. Although $b=1+p / q$ is rational, with $p$ odd, one may always choose $n_{+}$and $n_{-}$ so that $b$ is arbitrarily close to any given real number.

We have shown that $\operatorname{ISIM(2)}$ admits no deformations with de Sitter-like noncommutative translations. However, it is interesting to note that $\operatorname{ISIM(2),~unlike~the~Poincare~}$ group but like the Galilei group, admits a central extension: the cohomology group $H^{2}(\mathfrak{i} \mathfrak{i n}(2), \mathbb{R})$ is nontrivial. We find that it is generated by $\lambda^{+-} \wedge \lambda^{12}$, and so may adjoin to isim(2) a central element $Z$, whose only nontrivial Lie bracket is

$$
[N, J]=Z \text {. }
$$

Thus unlike the translations, the boosts and rotations can be rendered noncommutative. This also works for the full 2-parameter family of deformations of ISIM(2). Including the extra generator $Z$, appending $e^{\theta Z}$, and proceeding with the construction of an invariant particle Lagrangian leads to unmodified equations of motion, since the only effect is to add a total derivative $\dot{\theta}$ to the Lagrangian.

It was argued in [13] that æther-drift experiments imply $|b|<10^{-10}$. However, it follows from (17) that every particle has a mass tensor $m_{i j}=(1-b) m\left(\delta_{i j}+b n_{i} n_{j}\right)$. Hughes-Drever-type limits [17] on the anisotropy of inertia then potentially imply that $|b|<10^{-26}$. However, this depends on the precise form of the interactions $[18,19]$. Since a nonvanishing $b$ could arise through quantum corrections, very special relativity faces the question, analogous to the puzzle posed by the cosmological constant in traditional relativity: "Why is $b$ so small?"

We are grateful to Dan Freedman and Jaume Gomis for helpful discussions, and to the Galileo Galilei Institute in Florence, the workshop on String and M-Theory Approaches to Particle Physics and Cosmology, and the INFN, for support and hospitality during the course of this work. We thank George Bogoslovsky for a helpful discussion about upper bounds on the parameter $b$. This work has also been supported by the European EC-RTN project MRTN-CT-2004-005104, MCYT FPA 2004-04582-C0201, CIRIT GC 2005SGR-00564. The research of C.P. was supported in part by DOE Grant No. DE-FG0395ER40917.
[1] A. G. Cohen and S. L. Glashow, Phys. Rev. Lett. 97, 021601 (2006).

[2] We take the Minkowski metric to be $d s^{2}=\eta_{\mu \nu} d x^{\mu} d x^{\nu}=$ $2 d x^{+} d x^{-}+d x^{i} d x^{i}$.

[3] Although not named, ISIM(2) appears to have been first introduced in J. B. Kogut and D. E. Soper, Phys. Rev. D 1,
2901 (1970)

[4] P. A. M. Dirac, Nature (London) 168, 906 (1951); 169, 146 (1952); Sci. Mon. 78, 142 (1954).

[5] A. Dunn and T. Mehen, arXiv:hep-ph/0610202.

[6] A. G. Cohen and D. Z. Freedman, J. High Energy Phys. 07 (2007) 039. 
[7] U. Lindström and M. Roček, arXiv:hep-th/0606093.

[8] G.W. Gibbons, J. Gomis, and C. N. Pope (work in progress).

[9] D.Z. Freedman (private communication).

[10] J. Patera, R. T. Sharp, P. Winternitz, and H. Zassenhaus, J. Math. Phys. (N.Y.) 18, 2259 (1977).

[11] M. Levy-Nahas, J. Math. Phys. (N.Y.) 8, 1211 (1967).

[12] S. R. Coleman, J. Wess, and B. Zumino, Phys. Rev. 177, 2239 (1969).

[13] G. Bogoslovsky, arXiv:0706.2621.

[14] J. Gomis, K. Kamimura, and P. West, Classical Quantum
Gravity 23, 7369 (2006).

[15] A. J. Hariton and R. Lehnert, Phys. Lett. A 367, 11 (2007).

[16] G. Y. Bogoslovsky and H. F. Goenner, Phys. Lett. A 323, 40 (2004).

[17] S. K. Lamoreaux et al., Phys. Rev. Lett. 57, 3125 (1986); T. E. Chupp et al., Phys. Rev. Lett. 63, 1541 (1989).

[18] G. Y. Bogoslovsky, Nuovo Cimento Soc. Ital. Fis. B 77, 181 (1983).

[19] J. Fan, W. D. Goldberger, and W. Skiba, Phys. Lett. B 649, 186 (2007). 